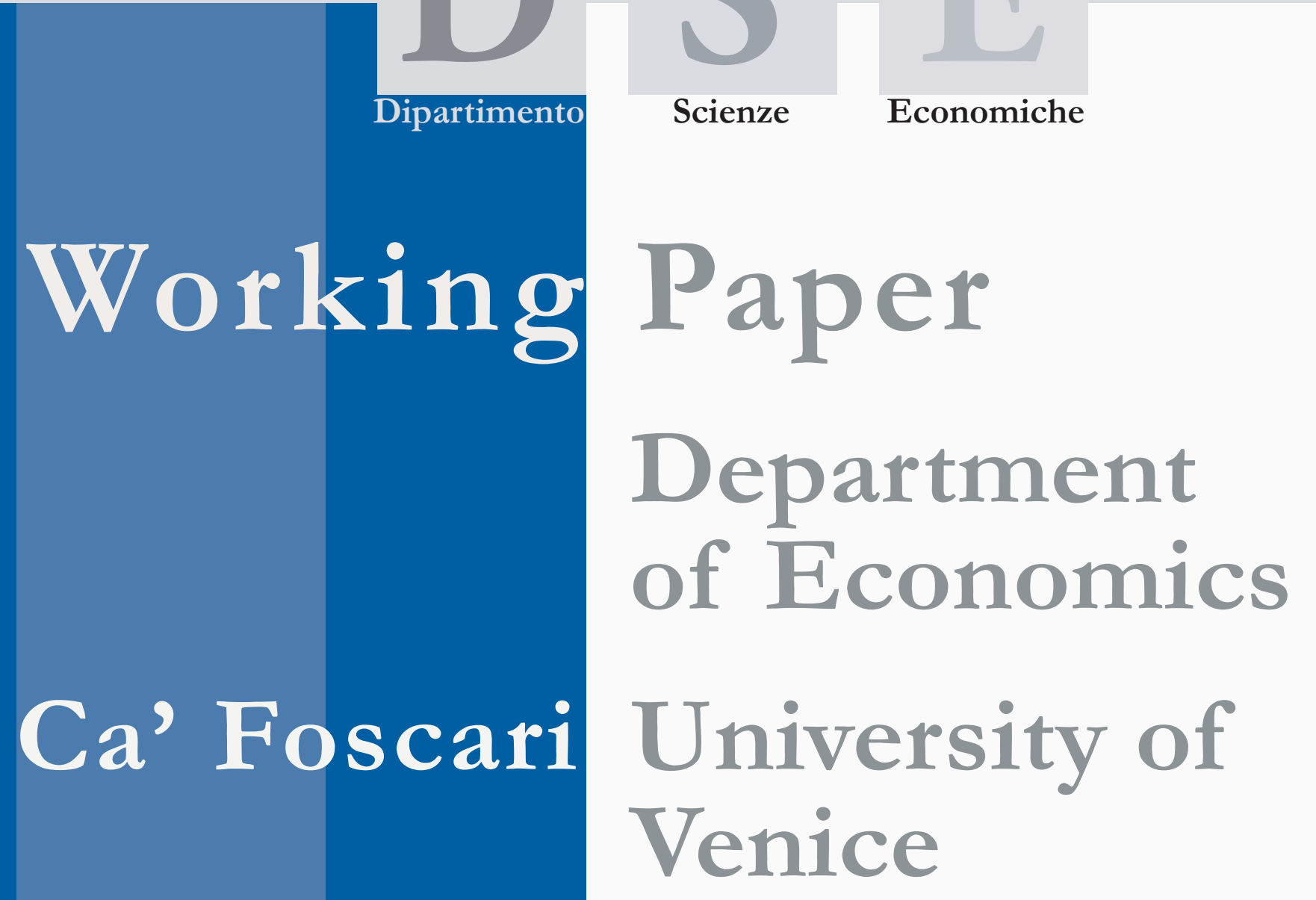

Sergio Currarini

Francesco Feri

Information Sharing

Networks in Oligopoly 


\title{
Information Sharing Networks in Oligopoly
}

\author{
Sergio Currarini \\ Università di Venezia
}

\section{Francesco Feri}

Università di Innsbruck

First Draft: June 2008

\begin{abstract}
We study the incentives of oligopolistic firms to share private information on demand parameters. Differently from previous studies, we consider bilateral sharing agreements, by which firms commit at the ex-ante stage to truthfully share information. We show that if signals are i.i.d., then pairwise stable networks of sharing agreements are either empty or made of fully connected components of increasing size. When linking is costly, non complete components may emerge, and components with larger size are less densly connected than components with smaller size. When signals have different variances, incomplete and irregular network can be stable, with firms observing high variance signals acting as "critical nodes". Finally, when signals are correlated, the empty network may not be pairwise stable when the number of firms and/or correlation are large enough.
\end{abstract}

\section{Keywords}

Information sharing, oligopoly, networks, Bayesian equilibrium

\section{JEL Codes}

D43, D82, D85, L13

\author{
Address for correspondence: \\ Sergio Currarini \\ Department of Economics \\ Ca’ Foscari University of Venice \\ Cannaregio 873, Fondamenta S.Giobbe \\ 30121 Venezia - Italy \\ Phone: (++39) 0412349133 \\ Fax: (++39) 0412349176 \\ e-mail: s.currarini@unive.it
}

This Working Paper is published under the auspices of the Department of Economics of the Ca' Foscari University of Venice. Opinions expressed herein are those of the authors and not those of the Department. The Working Paper series is designed to divulge preliminary or incomplete work, circulated to favour discussion and comments. Citation of this paper should consider its provisional character.

The Working Paper Series
is availble only on line
(www.dse.unive.it/pubblicazioni)
For editorial correspondence, please contact:
wp.dse@unive.it

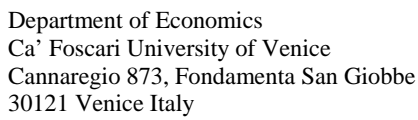




\section{Introduction}

The incentives of oligopolistic firms to share private information about a random demand intercept have been studied by vast literature, pioneered by the works of Novshek and Sonnenschein (1982), Clarke (1983), Vives (1985), Li (1985) and Gal-or (1985). All works in this literature has modeled the transmission of information by assuming that firms disclose their private information to a central agency (e.g., a Trade Association), which aggregates all the received information and delivers it back to firms. While in most papers it is assumed that firms receive the aggregated information independently of their disclosure decision, some authors have studied a quid pro quo exchange mechanism, in which firms receive the aggregated information if and only if they have disclosed their own (see Clark (1983), Kirby (1988), Raith (1996)).

In the case of quid pro quo exchange, most results have compared firms' expected profits in a scenario of complete absence of information with profits in a scenario in which all firms share their own information. Kirby (1988) has studied the Nash Equilibrium of a game in which each firm decides its disclosure behaviour in a quid pro quo exchange,game.

In this paper we contribute to this literature by exploring the equilibrium sharing behaviour of firms when contracts are bilateral. In our model, firms meet in pairs, and decide whether to share their own private information by means of an quid pro quo contract. What we have in mind is therefore not a central device of information transmission, such as a Trade Association, but rather a series of private and bilateral contacts, by which firms share information in a targeted way, without necessarily disclosing it to all firms in the system. The structure of bilateral sharing agreement is well represented by a non directed network, in which nodes represent firms, and links represent agreements.

In this new context we address old and new questions in a simple Cournot game with homogeneous goods and no production costs. Do we observe information sharing in equilibrium? Can we characterize the equilibrium architectures agreements? Can we trace the sharing behaviour of firms to their position in the network and vice versa? And what is the role of asymmetry and correlation of private signals in determining equilibrium networks?

We employ the model of Gal-Or (1985), in which a set of Cournot oligopolists each observes a piece of the demand intercept. In this framework, the signals observed by firms can be independently distributed, thus allowing for a benchmark model in which the model remains tractable. For the case of i.i.d signals, we are able to fully characterize the set 
pairwise stable sharing networks. ${ }^{1}$ In this simple case, the incentives of two firms to share information turn out to only depend on their relative degrees in the network (that is, on the number of firms that observe their signals). In particular, the incentive of firm $i$ to share information with firm $j$ increase with the degree of $i$ and decrease with the degree of $j$. Information sharing presents here clear aspects of congestion, with signals observed by many firms having less informational value than signals viewed by few. One way of explaining this result is the following. Expected profits are strictly related to the variability of firms equilibrium strategies in the Cournot game. The acquisition of new information has the effect of adding one source of variability (the new observed signal) and to reduce the variability of the signals already observed (by sharing it with another firm). The variability added by the acquisition of the new signal decreases with the number of firms observing that signal (because of the larger correlation of firms' equilibrium strategies), while the loss in variability due to the disclosure of one's own signal decreases with the number of firms that already observe that signal.

This simple structure of incentives originate a large set of pairwise stable networks with, nonetheless, neat qualitative features. First, the empty network (with no information sharing) is always stable, a result that echoes previous literature. Indeed, two isolated firms have no incentives to share information, just like two duopolist in the traditional approach. Second, there exist many other pairwise stable networks in which positive information is shared, all made of completely connected components with strictly increasing size. Firms are therefore organized in "Information Sharing Groups", each informationally equivalent to a centralized transmission device. For this benchmark model, we also look at the effect of an exogenous linking cost. Somewhat surprisingly, a small cost lead to qualitative differences in the incentives to share and in equilibrium information structures. In particular, non complete components are now possible as part of a stable network. Moreover, the "density" of a component (measure as the ratio between its average degree and its size) decreases with its size. In other words, small groups of firms share information more tightly that large groups.

We then study extensions of this benchmark model. We first allow independent signals to have heterogeneous variances. The incentives to form a link depend now on the relative degrees of firms and on the relative variance of their private signals. In general, the incentive

\footnotetext{
${ }^{1}$ The well known concept of pairwise stability, first used in Jackson and Wolinsky (1996)), requires that no link is either added or severed from the network. Adding a link requires mutual consent, while severing requires the conent of one firm only.
} 
of firm $i$ to form a link with firm $j$ are decreasing in the variance of the signal observed by $i$, and increasing in the variance of $j$ 's signal. Intuitively, a large variance signal has a large informational value, since it allows equilibrium strategies to acquire more variability. Although the empty network remains an equilibrium, new and informationally incomplete architecture are now possible. In particular, we show that non regular networks, usually referred to as "core-periphery", are stable, in which high variance firms have high degree and act as "connectors", indirectly linking low variance firms.

We finally turn to the richer and more complex case of correlated signals. We first show that the empty network is not be pairwise stable for high enough levels of correlation. In such cases, some positive amount of information sharing is present in all pairwise stable networks - a result to be contrasted with the traditional view that information sharing is incompatible with equilibrium in Cournot games with homogeneous goods. Intuitively, the additional incentives of two firms to share information come from the improved inference over the signals observed by the remaining firms; this better inference comes without any additional disclosure of information to the remaining firms and is, in this sense, at no cost. After showing that the complete network is always stable, we fully work out the case of four firms, for which we characterize the set of stable network for different ranges of the correlation parameter. It is of some interest to analyze the effect of correlation on the incentives to share. In particular, as correlation grows we pass from a stable structure in which one isolated firm refuses to join the information sharing group formed by the other firms, to one in which the same firm is excluded from the group. Intuitively, for low levels of correlation, the isolated firm finds it profitable to exploit the full variance of its own signal; as correlation increases, the profitability for the isolated firm of observing another signal increases, simply because of the increased inference power of such signal. However, the high level of correlation allows the sharing group to have a good enough inference on the isolated firm, which is therefore excluded.

We start by presenting the basic model, the information sharing mechanism and the Cournot game played by firms. In section 3 we study the set of pairwise stable networks when signals are independently distributed. Section 4 studies the case of correlated signals. Section 5 concludes the paper. 


\section{The Model}

\section{$2.1 \quad$ Set-up}

We consider a $n$-firms oligopolistic market with linear demand and no costs. The size of the market, given by the intercept of the demand function, is the sum of $n$ random variables $\left(a_{1}, a_{2}, \ldots, a_{n}\right)$ plus a deterministic part $A$ :

$$
p(Q)=A+a-b Q
$$

where $Q \equiv \sum_{i=1}^{n} q_{i}$ is the aggregate produced level in the market, and where $a \equiv a_{1}+a_{2}+\ldots+a_{n}$. Each variable $a_{i}$ is private information of firm $i$.

The vector of random variables $\left(a_{1}, a_{2}, \ldots, a_{n}\right)$ is jointly normally distributed with zero mean and matrix of variances and covariances:

$$
\Omega=\left[\begin{array}{cccc}
\sigma_{11} & \sigma_{12} & \ldots & \sigma_{1 n} \\
\ldots & \sigma_{22} & \ldots & \sigma_{2 n} \\
\ldots & \ldots & \ldots & \ldots \\
\ldots & \ldots & \ldots & \sigma_{n n}
\end{array}\right] .
$$

\section{$2.2 \quad$ Information Sharing}

Before observing the realization of private signals, firms can engage in bilateral information sharing agreements. By signing such an agreement, firms $i$ and $j$ commit to truthfully exchange the private information they receive, so that firm $i$ gets to observe the realization of the random variable $a_{j}$ and viceversa.

Bilateral agreements need not be transitive: the agreements $i j$ and $j k$ need not imply the existence of an agreement between firms $i$ and $k$. This is the main difference between the present approach and the previous literature, in which the transmission of a firm's private signals has a universal nature, as it is immediately and non exclusively received by all other firms.

Bilateral sharing agreements generate an information structure that can be usefully described as a non directed network. Given a set $N$, a non directed network $g$ is defined as any subset of the set of all (unordered) pairs of elements in $N$ :

$$
g \subseteq\{i j: i \in N \text { and } j \in N, i \neq j\}
$$


The elements of $N$ are called nodes, and a pair $i j \in g$ is called a "link". We denote by $N_{i}^{g}=\{j \in N: i j \in g\} \cup\{i\}$ the neighbourhood of $i$ in $g$, with cardinality $n_{i}^{g}$ (this cardinality is called "degree" of $i$ ). We will denote by $g-i j$ the network obtained from $g$ by deleting the link $i j \in g$ and by $g+i j$ the network obtained from $g$ by adding the link $i j \notin g$.

The network $g$ is connected if for all pairs $i$ and $j$ in $N$ there exists a connecting path $P(i, j)$, that is, a set $\left\{i_{1}, i_{2}, \ldots, i_{k}\right\}$ such that $i=i_{1}, j=i_{k}$, and $i_{p} i_{p+1} \in g$ for all $p=1, \ldots, k-1$. Given the network $g$, the network $h$ with set of nodes $M \subseteq N$ is a subnetwork of $g$ if $h \subseteq g$. The subnetwork $h$ of $g$ is a component of $g$ if it is connected and if for all $i \in M$ and $j \notin M$ we have $i j \notin g$. A component $h$ is regular if all $i \in h$ have the same degree. With some abuse of terminology, we will say that the network $g$ is regular when all its components are regular. The size of a component $h$, denoted by $n(h)$, is the cardinality of the set $M$. A component is completely connected is it is regular and its size coincides with its degree.

We derive the incentives of firms to form and sever links by associating with each possible network $g$ the expected profits of each firm $i$, denoted by $E\left[\pi_{i}(g)\right]$. These expectations are taken ex-ante, as decisions to sign sharing agreements are made before the realization of private signals. ${ }^{2}$ In more detail, once private signals are realized, firms play the Cournot game with incomplete information $\Gamma^{g}$, in which the information available to firm $i$ is given by the vector $a_{i}^{g}=\left\{a_{j}: j \in N_{i}^{g}\right\}$. We will denote by $E\left[\cdot \mid a_{i}^{g}\right]$ the expectation conditional on the information $a_{i}^{g}$, and by $E_{i}^{g}[\cdot]$ the expectation taken over all possible realization of the signals $a_{i}^{g}$. Finally, we denote by $q_{i}(g)$ the quantity set by firm $i$ in the Bayesian Nash Equilibrium of the game $\Gamma^{g}$. We have the following standard result (see proposition 1 in Kirby (1988)).

Lemma 1 Ex-ante expected profits of firm $i$ in the game $\Gamma^{g}$ are given by:

$$
E\left[\pi_{i}(g)\right]=E_{i}^{g}\left[b q_{i}(g)^{2}\right]
$$

Following Jackson and Wolinsky (1996), we use the concept of pairwise stable networks as a solution concept for the network formation stage. The network $g$ is pairwise stable if no link is either formed or severed.

Definition 1 The network $g$ is pairwise stable iff:

\footnotetext{
${ }^{2}$ In this we follow most of the existing literature. A study of link formation at the interim stage is of great interest, and involves strategic consideration and technical difficulties that are not present in the ex-ante approach. This is left for future research.
} 
i) $E\left[\pi_{i}(g)\right]-E\left[\pi_{i}(g-i j)\right] \geq 0$ and $E\left[\pi_{j}(g)\right]-E\left[\pi_{j}(g-i j)\right] \geq 0$ for all $i j \in g$;

ii) if $E\left[\pi_{i}(g+i j)\right]-E\left[\pi_{i}(g)\right]>0$ then $E\left[\pi_{i}(g+i j)\right]-E\left[\pi_{j}(g)\right]<0$, for all $i j \notin g$.

\subsection{Bayesian Cournot Equilibrium for a Given Network of Agreements}

Before turning to the analysis of pairwise stable networks in the next sections, we study the structure of Bayesian Nash Equilibrium of the game $\Gamma^{g}$ in more detail. A pure strategy for firm $i$ is a function $s_{i}: R_{+}^{n_{i}^{g}} \rightarrow R_{+}$setting a quantity level for each possible vector of signal observed by $i$. A Bayesian Nash equilibrium of this game is a profile of strategies $\left(s_{i}\right)_{i=1, \ldots, n}$ such that for all $i$, for all $q_{i}$ and for all $a_{i}^{g}$ :

$$
\begin{gathered}
E_{i}\left[\left(A+a-b\left(s_{i}\left(a_{i}^{g}\right)+\sum_{j \neq i} s_{j}\left(a_{j}^{g}\right)\right)\right) s_{i}\left(a_{i}^{g}\right) \mid a_{i}^{g}\right] \geq \\
\geq E_{i}\left[\left(A+a-b\left(q_{i}+\sum_{j \neq i} s_{j}\left(a_{j}^{g}\right)\right)\right) q_{i} \mid a_{i}^{g}\right]
\end{gathered}
$$

The first order conditions (one for each $i \in N$ ) that characterize a Bayesian Nash equilibrium for the game $\Gamma^{g}$ is given by

$$
q_{i}=\frac{A+\sum_{j \in N_{i}^{g}} a_{j}+E_{i}\left[\sum_{j \notin N_{i}^{g}} a_{j} \mid a_{i}^{g}\right]-b E_{i}\left[\sum_{j \neq i} s_{j}\left(a_{j}^{g}\right) \mid a_{i}^{g}\right]}{2 b}
$$

Applying standard results on linear Cournot games with incomplete information (see Radner (1962)), we can work with equilibrium strategies that are affine in signals. Let $\alpha_{i}$ be the constant term of the equilibrium strategy of firm $i$, and $\beta_{i j}$ be the coefficient applied by firm $i$ to each element $a_{j}$ of the vector $a_{i}^{g}$. To identify the equilibrium parameters, we substitute in (3) the affine expression of the strategies of players other than $i$ :

$$
q_{i}=\frac{A+\sum_{j \in N_{i}^{g}} a_{j}+\sum_{j \notin N_{i}^{g}} E_{i}^{g}\left[a_{j}\right]-b\left[\sum_{j \neq i} \alpha_{j}+\sum_{j \neq i}\left(\sum_{k \in N_{j}^{g} \backslash N_{i}^{g}} \beta_{j k} E_{i}\left[a_{k} \mid a_{i}^{g}\right]+\sum_{k \in N_{j}^{g} \cap N_{i}^{g}} \beta_{j k} a_{k}\right)\right]}{2 b} .
$$

We can explicitly derive the expectation of firm $i$ over firm $j$ 's signal (for $j \notin N_{i}^{g}$ ) by applying standard Bayesian updating formulae:

$$
E_{i}\left[a_{j} \mid a_{i}^{g}\right]=\Sigma_{i j}^{g} \Sigma_{i}^{-1} a_{i}^{g}=\sum_{h \in N_{i}^{g}} \lambda_{h j}^{i}(g) a_{h},
$$


where $\Sigma_{i j}^{g}$ is the $\left(1 \times n_{i}^{g}\right)$ vector of covariances between the signals in $a_{i}^{g}$ and signal $a_{j}, \Sigma_{i}$ is the $\left(n_{i}^{g} \times n_{i}^{g}\right)$ matrix of variances-covariances of the signals in $a_{i}^{g}$ and $\lambda_{h j}^{i}(g)$ is the updating coefficient applied by agent $i$ to signal $a_{h}$ to infer signal $a_{j}$ in network $g$ (we discuss the expression of $\lambda$ coefficients in some detail in section 4).

Using (3) and (5) we obtain an expression of $q_{i}$ in terms only of the signals observed by firm $i$, that we can use to identify the parameters of $i$ 's linear equilibrium strategy:

$$
\begin{aligned}
& q_{i}=\frac{A+\sum_{j \in N_{i}^{g}} a_{j}+\sum_{k \notin N_{i}^{g}} \sum_{j \in N_{i}^{g}} \lambda_{j k}^{i}(g) a_{j}}{2 b}- \\
& \frac{b\left[\sum_{h \neq i} \alpha_{h}+\sum_{h \neq i}\left(\sum_{k \in N_{h}^{g} \backslash N_{i}^{g}} \beta_{h k} \sum_{j \in N_{i}^{g}} \lambda_{j k}^{i}(g) a_{j}+\sum_{j \in N_{h}^{g} \cap N_{i}^{g}} \beta_{h j} a_{j}\right)\right]}{2 b} .
\end{aligned}
$$

Rearranging terms we obtain an expression for the equilibrium parameters of $i$ 's strategy:

$$
\begin{aligned}
\beta_{i j} & =\frac{1+\sum_{k \notin N_{i}^{g}} \lambda_{j k}^{i}(g)-b\left[\sum_{h \neq i} \sum_{k \in N_{h}^{g} \backslash N_{i}^{g}} \beta_{h k} \lambda_{j k}^{i}(g)+\sum_{h \in N_{j}^{g} \backslash\{i\}} \beta_{h j}\right]}{2 b}, \forall j \in N_{i}^{g} ; \\
\alpha_{i} & =\frac{A-b \sum_{h \neq i} \alpha_{h}}{2 b} .
\end{aligned}
$$

We first note that the $\alpha$ parameters are defined by an independent set of equations, and that they do not depend on the network $g$. Since the expected values of all signal is zero, this implies that the expected aggregate quantity is the same in each network.

We then observe that the expression for the terms $\beta$ has intuitive interpretations that highlight the role of the network in shaping equilibrium behaviour. The term $\beta_{i j}$ (measuring the reaction of firm $i$ to the observed signal $a_{j}$ ) is determined by the use that firm $i$ makes of signal $a_{j}$ to estimate the intercept (the term $1+\sum_{k \notin N_{i}^{g}} \lambda_{j k}^{i}(g)$ ), minus the two terms in the squared brackets. The first measures the use that firm $i$ makes of signal $a_{j}$ in order to estimate the reaction of the other firms to signals that $i$ does not observe. If signals are positively correlated, the larger the terms $\lambda_{j k}^{i}(g)$ (more correlated signals), the less firm $i$ should react to signal $a_{j}$. This is due to the facts that $a_{j}$ predicts the behaviour of the other firms and that strategies are substitutes. The second term has a different, though related, interpretation. Firm $i$ reacts less to signal $a_{j}$ the more numerous are the firms that observe 
$a_{j}$ and base their decisions on it. Intuitively, the information provided by signal $a_{j}$ is more valuable the less it is observed by other firms - a clear congestion effect.

\section{Pairwise Stable Networks with Independently Distributed Signals}

If signals are independently distributed, the matrix $\Omega$ has all zeros outside the main diagonal. For this case, the equilibrium parameters of firm $i$ are (see (6)-(7)):

$$
\begin{aligned}
\beta_{i j} & =\frac{1-b \sum_{h \in N_{j}^{g} \backslash\{i\}} \beta_{h j}}{2 b}, \forall j \in N_{i}^{g} ; \\
\alpha_{i} & =\frac{A-b \sum_{h \neq i} \alpha_{h}}{2 b} .
\end{aligned}
$$

From (8) we note that for each $j$, the equilibrium coefficients applied to signal $a_{j}$ by the firms in $N_{j}^{g}$ are determined by an independent set of identical equations, so that $\beta_{i j}=\beta_{h j}$ for all $i$ and $h$ in $N_{j}^{g}$. Also, from (9) we note that the coefficients $\alpha_{i}$ are constant with respect to the network $g$, and that $\alpha_{i}=\alpha_{h}$ for all $i$ and $h$.

\subsection{Identically Distributed Signals}

This section presents the benchmark model of i.i.d. signals, for which all terms on the main diagonal of matrix $\Omega$ are equal, that is $\sigma_{i i}=\sigma_{j j}=\sigma$ for all $i, j \in N$.. For this case we are able to fully characterize the set of pairwise stable networks by exploiting the extremely simple structure of incentives. We start by expressing necessary and sufficient conditions for a network to be pairwise stable.

Proposition 1 A network $g$ is pairwise stable if and only both of the following conditions are verified.

For all $i j \in g$ :

$$
\begin{aligned}
& \frac{1}{\left(n_{j}^{g}+1\right)^{2}}+\frac{1}{\left(n_{i}^{g}+1\right)^{2}} \geq \frac{1}{\left(n_{i}^{g}\right)^{2}} \\
& \frac{1}{\left(n_{j}^{g}+1\right)^{2}}+\frac{1}{\left(n_{i}^{g}+1\right)^{2}} \geq \frac{1}{\left(n_{j}^{g}\right)^{2}} .
\end{aligned}
$$


For all $i j \notin g$ :

$$
\begin{aligned}
\frac{1}{\left(2+n_{i}^{g}\right)^{2}}+\frac{1}{\left(2+n_{j}^{g}\right)^{2}} & >\frac{1}{\left(1+n_{i}^{g}\right)^{2}} \\
\text { implies } \frac{1}{\left(2+n_{i}^{g}\right)^{2}}+\frac{1}{\left(2+n_{j}^{g}\right)^{2}} & <\frac{1}{\left(1+n_{j}^{g}\right)^{2}} .
\end{aligned}
$$

The proof this and all other results of the paper can be found in the appendix.

Condition (10) requires that no link in a stable network is severed: on the LHS is the gain in terms of $i$ 's equilibrium quantity's variance due to the maintenance of link $i j$, which has to be greater then the cost of maintaining the link. Condition (11) requires the same for $j$. Conditions (12-13) requires that no link is added to a stable network. If the net gain for firm $i$ of forming the link $i j$ exceeds the cost $c$, then the reverse must hold for firm $j$.

The incentives to form or sever a link $i j$ only depend on the degree of the nodes $i$ and $j$ in the network. This is a direct implication of the separability in signals of the equilibrium coefficients $\beta$ : the formation or severance of the link $i j$ does not affect the way in which firms react to all signals other than $a_{i}$ and $a_{j}$. The $\beta$ coefficients determine the variability of equilibrium strategies and, as shown in the proof of proposition 1, the level of expected profits (note here that by the assumption of independent signals, the total variance of equilibrium quantities is separable across signals). So, although total expected profits will typically vary in different networks, the difference in expected profits induced by the formation of the link $i j$ in the network $g$ will be the same as in the network $g^{\prime}$ as long as $n_{i}^{g}=n_{i}^{g^{\prime}}$ and $n_{j}^{g}=n_{j}^{g^{\prime}}$. Note that the effect of degrees on incentives described in proposition 1 are similar to those at work in the coauthor model studied by Jackson and Wolinsky (1996). As in that model, here the incentives of a firm $i$ to link with another firm $j$ decrease with the $j$ 's degree and increase with $i$ 's degree.

We are therefore able to identify thresholds in the degree of a node $j$, below which a node $i$ of a given degree would be willing to form (or not to sever) the link $i j$.

Definition 2 For any $n_{i}^{g}$, the threshold $F\left(n_{i}^{g}\right)$ has the property that firm $i$ does not sever a link with firm $j$ (condition 10 is satisfied) if and only if $n_{j}^{g} \leq F\left(n_{i}^{g}\right)$.

Definition 3 For any $n_{i}^{g}$, the threshold $f\left(n_{i}^{g}\right)$ has the property that firm $i$ wishes to form a link with firm $j$ (condition 12 is satisfied) if and only if $n_{j}^{g}<f\left(n_{i}^{g}\right)$. 
It can be easily shown that $F(n)$ and $f(n)$ exist for all $n$. In particular we have:

$$
\begin{aligned}
F(n) & =\sqrt{\frac{n^{2}(1+n)^{2}}{1+2 n}}-1 ; \\
f(n) & =\sqrt{\frac{(n+1)^{2}(2+n)^{2}}{2 n+3}}-2 .
\end{aligned}
$$

We start the analysis of stable pairwise networks by recording properties of the threshold functions $F$ and $f$, that will be exploited in the propositions to follow.

Lemma 2 i) $F(n)>F(m)$ if $n>m$.

ii) $f(n)>f(m)$ if $n>m$;

iii) $F(n)-1=f(n-1)$.

iv) $F(n)-F(n-1)>1$.

v) $n \leq f(n)$ if and only if $n \geq 2$ and $n \leq F(n)$ if and only if $n \geq 3$.

vi) $F(n)<f(n)$ for all $n \geq 2$.

Properties i) and ii) establish monotonicity of $F$ and $f$ : the larger the degree of a node $i$, the larger the maximal degree of the nodes that $i$ is willing to stay linked to and to form a new link with. Property iii) simply says that if a node with degree $n$ is not severing a link with a node with degree $m$, then a node with degree $n-1$ is willing to form a link with a node of degree $m-1$. Property iv) is purely technical. Property $v$ ) says that isolated nodes have no incentives to link; in all other cases, nodes with equal degree have an incentive to stay linked. Finally, property vi) implies that if a node of degree $n$ is linked with a node of degree $m$, then it has an incentive to link with all other nodes of degree $m$ in the network.

The next proposition characterizes the set of pairwise stable networks for the case of i.i.d. signals.

Proposition 2 Let $n \geq 3$. The set of pairwise stable networks is characterized by the following properties.

i) Every pairwise consists of $p \geq 1$ completely connected components, with size either 1 (isolated nodes) or at least 3.

ii) Both $p=1$ (complete) and $p=n$ (empty) are always equilibrium architectures.

iii) All components with size of at least 3 are strictly ordered according to size. In particular, for all $S \subset N$, all architectures in which all nodes in $S$ appear has singleton and 
in which the set of nodes in $N \backslash S$ is organized in p completely connected components of size $n_{1}, n_{2}, \ldots, n_{p}$ such that $n_{i}>f\left(n_{i+1}\right)$ for all $i=1, \ldots, p-1$, are stable architectures.

The set of pairwise stable networks characterized in Proposition 2 is very large. However, we obtain two precise qualitative predictions on stable networks. First, information sharing is organized in groups (the fully connected components), within which the transmission of information is equivalent to one in firms publicly discloses its observed signal to all other firms in the group. Second, information sharing groups must be of different size, to make sure that firms in different groups do not form links (remember that by point $(v)$ in lemma 2 , firms with similar degree link together). For convenience, we will refer to such fully connected components as "Information Sharing Groups" (ISG), and to the architectures made only of such components as ISG networks.
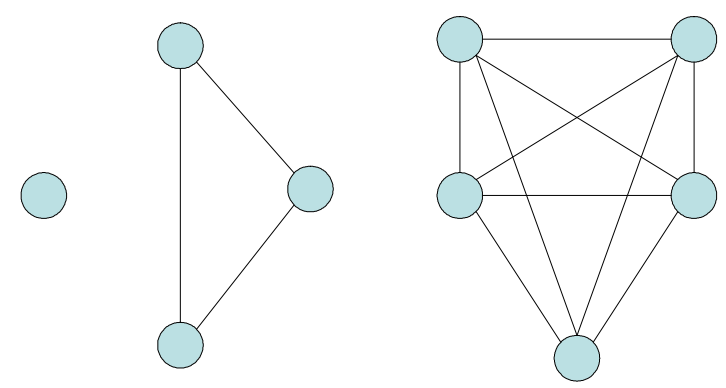

Figure 1. A Pairwise Stable ISG Network

We remark that the empty network is always a stable architecture, confirming the traditional results of absence of information sharing in Cournot Oligopoly with demand uncertainty. The stability of other regular structures is a result of the incentives to share information of non isolated firms with equal degree. Here we see the effect of the bilateral structure of contracts, and the implication of adopting a link-based concept of stability: the incentives of two oligopolists to share information crucially depend on their location in the network. 


\subsection{Signals with Heterogeneous Variances}

In this section we relax the assumption that signals are identically distributed, and allow the variances of firms' signals - the terms on the main diagonal of $\Omega$ - to differ. In this new context we are able to address new and interesting issues, relating the architecture of stable networks, and the position of various firms in such architectures, to the distribution of variances.

For simplicity we focus on the case of no linking costs. Steps at all similar to those employed in the proof of proposition 1 lead to the following necessary and sufficient conditions for a network $g$ to be pairwise stable:

For all $i j \in g$ :

$$
\begin{aligned}
& \frac{\sigma_{j j}}{\left(n_{j}^{g}+1\right)^{2}}+\frac{\sigma_{i i}}{\left(n_{i}^{g}+1\right)^{2}} \geq \frac{\sigma_{i i}}{\left(n_{i}^{g}\right)^{2}} \\
& \frac{\sigma_{j j}}{\left(n_{j}^{g}+1\right)^{2}}+\frac{\sigma_{i i}}{\left(n_{i}^{g}+1\right)^{2}} \geq \frac{\sigma_{j j}}{\left(n_{j}^{g}\right)^{2}} .
\end{aligned}
$$

For all $i j \notin g$ :

$$
\begin{aligned}
\frac{\sigma_{i i}}{\left(2+n_{i}^{g}\right)^{2}}+\frac{\sigma_{j j}}{\left(2+n_{j}^{g}\right)^{2}}>\frac{\sigma_{i i}}{\left(1+n_{i}^{g}\right)^{2}} & \text { implies } \frac{\sigma_{i i}}{\left(2+n_{i}^{g}\right)^{2}}+\frac{\sigma_{j j}}{\left(2+n_{j}^{g}\right)^{2}}<\frac{\sigma_{j j}}{\left(1+n_{j}^{g}\right)^{2}} .
\end{aligned}
$$

Condition (16)-(19) tell us that, given the degrees $n_{i}^{g}$ and $n_{j}^{g}$, the incentive of $i$ to sever the link $i j$ increases with the ratio of variances $\frac{\sigma_{i i}}{\sigma_{j j}}$. Conversely, the incentive of $i$ to form the link $i j$ decreases with $\frac{\sigma_{i i}}{\sigma_{j j}}$. This can be understood in terms of the additional variability of $i$ 's equilibrium quantity due to the link $i j$. The higher the term $\sigma_{j j}$, the higher the additional variability of $i$ 's quantity due to $i j$, and the higher the informational "value" of $j$ 's signal for firm $i$. Similarly, the higher the term $\sigma_{i i}$, the lower the incentive of firm $i$ to form the link $i j$; this because it is costly to share a signal with high variance with one additional firm. Again, a high value of $\sigma_{i i}$ reflects therefore a high informational value of $i$ 's signal. The effect of firms' degrees on the incentive to form a link are therefore enriched by the effect of signals' variances, so that a firm with low degree may now find it profitable to form a link with a firm with high degree if the latter has a relatively high variance.

We start by looking at two architectures, the empty and the complete, that were shown to be always stable in the case of i.i.d. signals without costs: the empty and the complete 
networks. We first show that the empty network is still a pairwise stable architecture, and that this results holds true even if the firm with lower variance were allowed to make ex-ante transfers to convince a potential partner to sign a sharing agreement.

Proposition 3 The empty network is pairwise stable for all distributions of variances. Moreover, the empty network remains pairwise stable even if firms are allowed to make side payments at the ex-ante stage.

Differently from the symmetric case, the complete network fails to be stable when variances differ substantially. This comes as a result of the weak incentives of firms with high variance to link with firms with low variance (see conditions (16)-(17) and the subsequent discussion).

Example 1 Consider the complete network $g$ with three nodes. Node 1 has variance $\sigma_{1}=1$, while node 2 has variance $\sigma_{2}=\frac{7}{10}$. Condition (16) is not satisfied for node 1 who severs link 12.

In the example, link 12 is severed due to the large difference in the variances of firm 1's and 2's signals. From (16), the condition for firm $i$ to maintain the link $i j$ in the complete network $g$ is:

$$
\frac{\sigma_{i i}}{\sigma_{j j}} \leq \frac{n^{2}}{1+2 n}
$$

Although this condition is violated in example 1, we note that the RHS of (20) grows without bound with $n$, implying that (20) would be satisfied for $n$ large enough. This implies the following proposition.

Proposition 4 For any distribution of the variances, there exists a finite number of firms $\bar{n}$ such that for all $n \geq \bar{n}$ the complete network is pairwise stable.

We now turn to the effect of heterogeneity on the set of pairwise stable ISG networks other than the empty and complete ones. We first note that components with equal size may, in principle, be consistent with stability. In such structures, stability would require firms in different components to have different variances, in order not to face incentives to "bridge" components. This intuitive argument is made clear in the next proposition. Let $\left(\sigma_{11}, \sigma_{22}, \ldots, \sigma_{n n}\right)$ be the variances of the $n$ firms, with the convention that $\sigma_{i i}<\sigma_{j j}$ for all 
$i=1, \ldots, n-1$ and $j=i+1$. We say that the network $g$ is consecutive if components can be ordered as $\left(h_{1}, h_{2}, \ldots, h_{p}\right)$ with the following property. Let $\sigma_{-}(h)$ and $\sigma_{+}(h)$ be the lowest and highest variances of firms in component $h$. Then if $i \in h_{k+1}$ we have $\sigma_{i i}>\sigma_{+}(h)$ for all $k=1, \ldots, p-1$.

Proposition 5 Let $g$ be a pairwise stable network in which all components are fully connected and of the same size. Then $g$ is consecutive.

The next is an example of such stable structures.

Example 2 Consider a network with 9 nodes, made of three fully connected components of size 3, with set of nodes $h_{1}=\{12,23,13\}, h_{2}=\{45,56,46\}, h_{3}=\{78,89,79\}$. Variances are constant within components: $\sigma_{1}=\sigma_{2}=\sigma_{3}=1 ; \sigma_{4}=\sigma_{5}=\sigma_{6}=\frac{1}{2} ; \sigma_{7}=\sigma_{8}=\sigma_{9}=\frac{1}{4}$. For any link across components not to form we need the firm with larger variance $y$ not to wish to form the link with the firm with lower variance $x$ (see condition (19)):

$$
\frac{x}{25}+\frac{y}{25}<\frac{y}{16} \rightarrow x \leq \frac{225}{400} .
$$

The ratio $x=\frac{1}{2} y$ in the example satisfies this constraint for all components.

We finally turn to the analysis of pairwise stable networks which are not ISG networks. As for the case of i.i.d signals, we find that regular incomplete architecture are never pairwise stable.

Proposition 6 There exists no distribution of variances under which a pairwise stable network can include regular and incomplete components.

The result of Proposition 6 can be understood as follows. From the stability conditions derived in section 3.1, two firms with same degree and variance always have an incentive to form a link. We have also noted that if two firms have the same degree, then the firm with a higher variance has less incentive to form the link (see condition (18)). Proposition 6 shows that although we may eliminate the incentives of any two firms in a regular network to form a link by choosing the appropriate distribution of variances, by doing so we necessarily introduce incentives to sever links from the network. As a result, no incomplete and regular architecture is pairwise stable. 
Differently from the i.i.d. case, when firms have heterogeneous variance non regular architectures may be stable. Intuitively, this is due to the fact that firms with large variance and large degree need not have an incentive to sever a link, and, at the same time, can still be "attractive" for firms with lower variance and degree, acting therefore as "connectors" in the network. We show that such architectures, usually referred to as "core-periphery networks" can be pairwise stable for suitable distributions of variances. In more detail, core periphery networks present a dense set of interconnected nodes - the core - each linked with all nodes in the network, and sets of peripheral nodes which are internally connected and are linked with the core nodes (see figure 2). Formally, a core-periphery network $g$ consists of a set $\left\{g_{1}, g_{2}, \ldots . g_{H}\right\}$ of fully connected subnetworks, such that $i \in g_{k}$ and $j \in g_{m}$ implies that $i j \notin g$ for all $k \neq m$ such that $k=2,3, \ldots, H$ and $m=2,3, \ldots, H$, and such that $i \in g_{1}$ and $j \in g_{k}$ implies $i j \in g$ for all $k=1,2,3, \ldots, H$. We call the subnetwork $g_{1}$ core, and the subnetworks $\left\{g_{2}, \ldots . g_{H}\right\}$ peripheral planets. A symmetric core-periphery network is such that all peripheral planets contain the same number of nodes $n_{p} \geq 1$, and in which all nodes in the same planet have the same variance. The number of nodes in the core is denoted by $n_{c}$. We also have $H=\frac{n-n_{c}}{H}$.

Proposition 7 A symmetric core-periphery network is pairwise stable for some distribution of variances only if the following condition holds:

$$
\left(\frac{\left(n_{c}+n_{p}+1\right)^{2}}{2 n_{c}+2 n_{p}+3}\right)^{H-1} \leq \frac{n^{2}\left(n_{c}+n_{p}\right)^{2}}{(2 n+1)\left(2 n_{c}+2 n_{p}+1\right)} .
$$

A direct consequence of condition (21) is that the "star" network (in which $n_{p}=n_{c}=1$ ) is never pairwise stable. The next is an example of a stable core-periphery architecture.

Example 3 Consider a network with 5 nodes: node 1 is the "core" node, while the two peripheral components are $\{23\}$ and $\{45\}$. Variances are $\sigma_{1}=1, \sigma_{2}=\sigma_{3}=\frac{1}{5}, \sigma_{4}=\sigma_{5}=\frac{1}{2}$. Relevant stability conditions (16)-(19) (for links 12, 15 and 34, respectively) are satisfied:

$$
\frac{1}{36}+\frac{1}{5} \frac{1}{16} \geq \frac{1}{25} ; \frac{1}{36}+\frac{1}{2} \frac{1}{16} \geq \frac{1}{25} ;\left(\frac{1}{2}+\frac{1}{5}\right) \frac{1}{25} \leq \frac{1}{5} \frac{1}{36} .
$$




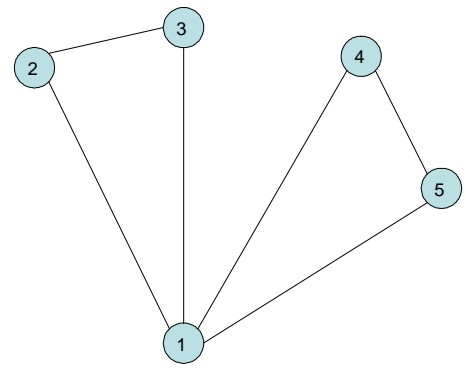

Figure 2. A core-periphery network

\subsection{The Effect of a Linking Cost}

Before turning to the more complex and richer case of correlated signals in section 4 , we briefly study the effect of an exogenous cost of forming links on the architecture of stable networks. The cost $c$ represents all monetary expenditures that a firm bears in order to arrange and execute an information sharing agreement. For instance, when such arrangements are viewed by the regulator as a signal of collusion, the cost $c$ may refer to the expected fine. For simplicity, and in order to isolate the pure effect of costs, we return here to the benchmark model of i.i.d. signals.

Interestingly, the addition of a linking cost has qualitative effects on the condition for pairwise stability, with a new role played by market conditions (the slope $b$ of the demand function) and by the magnitude of the variance $\sigma$. The following stability conditions are obtained by a minor modification of the proof of proposition 1 :

For all $i j \in g$ :

$$
\begin{aligned}
& \frac{1}{\left(n_{j}^{g}+1\right)^{2}}+\frac{1}{\left(n_{i}^{g}+1\right)^{2}}-\frac{1}{\left(n_{i}^{g}\right)^{2}} \geq \frac{b^{2} c}{\sigma} \\
& \frac{1}{\left(n_{j}^{g}+1\right)^{2}}+\frac{1}{\left(n_{i}^{g}+1\right)^{2}}-\frac{1}{\left(n_{j}^{g}\right)^{2}} \geq \frac{b^{2} c}{\sigma} .
\end{aligned}
$$


For all $i j \notin g$ :

$$
\begin{aligned}
\frac{1}{\left(2+n_{i}^{g}\right)^{2}}+\frac{1}{\left(2+n_{j}^{g}\right)^{2}}-\frac{1}{\left(1+n_{i}^{g}\right)^{2}} & >\frac{b^{2} c}{\sigma} \\
\operatorname{implies} & \frac{1}{\left(2+n_{i}^{g}\right)^{2}}+\frac{1}{\left(2+n_{j}^{g}\right)^{2}}-\frac{1}{\left(1+n_{j}^{g}\right)^{2}}<\frac{b^{2} c}{\sigma} .
\end{aligned}
$$

Notice the role played by the parameter $b$ : a more elastic demand (small $b$ ) provides higher incentives to maintain (and to form) links, while a rigid demand (large $b$ ) provides higher incentives to sever a link. From now on we will refer to the gross cost parameter $C=\frac{b^{2} c}{\sigma}$, and refer to the single elements of $C$ only when needed.

In the next proposition we study the effect of the new stability conditions on the set of pairwise stable networks. In order to compare the stable networks given a cost $c$ with those characterized in proposition 2 in the absence of costs, we restrict the analysis to the set of regular networks,

Proposition 8 Let $c>0$. There exist size levels $m_{1}(C) \leq 4 \leq m_{2}(C)$ such that all regular pairwise stable networks satisfy the following properties:

a) no component of size $2 \leq n(h)<m_{1}(C)$ exists.

b) all components $h$ of size $m_{1}(C) \leq n(h) \leq m_{2}(C)$ are fully connected;

c) all components $h$ of size $n(h) \geq m_{2}(C)$ have degree equal to $m_{2}(C)$;

We can better understand the effect of a linking cost $c$ on equilibrium architectures by first looking at stable ISG networks. Point $b$ ) imposes a lower bound and an upper bound on the size of a stable ISG (that is, of a stable fully connected component). These bounds can be understood as follows. The presence of a linking cost $c$ increases the incentives to sever links. This disrupts the stability of those components whose members have lower incentives to maintain their links (as these are expressed by the LHS of conditions (22)-(22)). As explained in the proof of Proposition 8, these incentives are non monotonic in the size $m$ of a component, increasing until $m=4$ and decrease thereafter. It follows that as the gross cost parameter $C$ increases, the largest and smallest ISGs are the first to drop out of the set of stable ISG. Interestingly, market conditions play a role for the size of ISGs. In particular, to more elastic demand curves correspond lower values of $C$, allowing for the emergence of large ISGs. 
The second effect of the cost $c$ is to decrease the incentives to form new links. This allows ISGs with similar size in a stable network, since links bridging components are harder to form.

Point $b$ ) of Proposition 8 considers the possibility that a pairwise stable network include non complete components. While the emergence of such structures is a natural consequence of a positive (large enough) cost $c$, proposition 8 provides information on the relation between the density of a component (measured here as the ration between average degree and size) and its size. In a stable configuration, larger groups partially share information (organizing in a non complete component), while smaller groups (with size smaller than $m_{2}(C)$ ) share information more intensely (see figure 3 ).

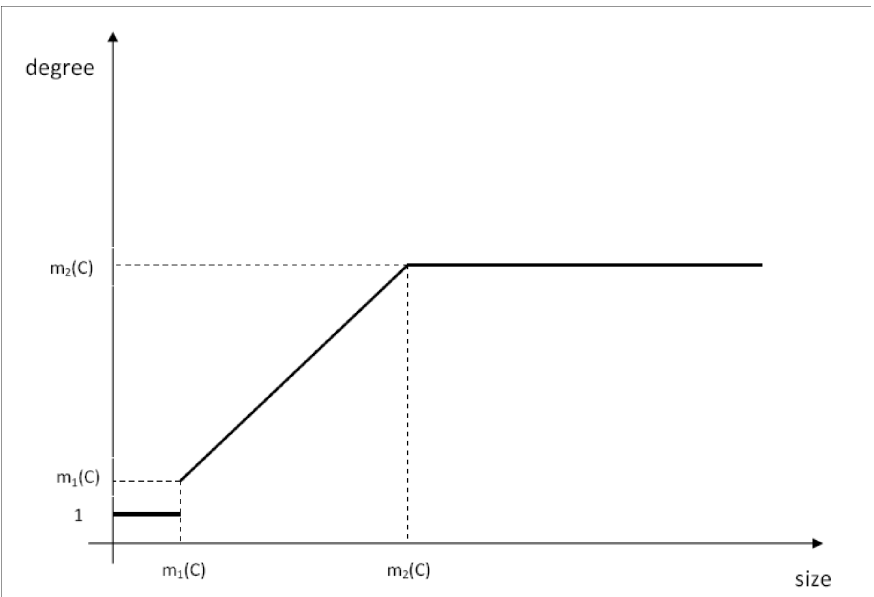

Figure 3. The relation between size and degree of stable components

\section{Stable Networks with Correlated Signals}

We finally turn to the case of correlated signal. For simplicity, we assume that signals are identically distributed. In this case the matrix $\Omega$ has a constant term $\sigma$ on the main diagonal, and a constant term $\rho \in[0, \sigma]$ elsewhere. The expression of the updating coefficients $\lambda$ introduced in condition (5) is as follows:

$$
\lambda_{h j}^{i}(g)=\lambda_{i}^{g}=\frac{\delta}{1+\delta\left(n_{i}^{g}-1\right)}, \forall h \in N_{i}^{g}, \forall j \notin N_{i}^{g},
$$


where $\delta \equiv \frac{\rho}{\sigma}$ is a correlation coefficient. We can now rewrite (6)-(7) as:

$$
\begin{aligned}
\beta_{i j} & =\frac{1+\left(n-n_{i}^{g}\right) \lambda_{i}^{g}-b\left[\lambda_{i}^{g} \sum_{h \neq i} \sum_{k \in N_{h}^{g} \backslash N_{i}^{g}} \beta_{h k}+\sum_{h \in N_{j}^{g} \backslash\{i\}} \beta_{h j}\right]}{2 b}, \forall j \in N_{i}^{g} ; \\
\alpha_{i} & =\frac{A-b \sum_{h \neq i} \alpha_{h}}{2 b} .
\end{aligned}
$$

Direct computations using (25)-(26) show that each firm prefers the empty network to the complete network for all possible values of the correlation parameter $\delta$. This is just a special case of proposition 4.4 in Raith (1996). However, the next proposition shows that empty network is not pairwise stable if correlation is larger that a given threshold.

Proposition 9 Let $n \geqslant 4$ and $\delta \in[0,1]$. There exists a threshold $\bar{\delta}(n)$ such that for all $\delta \geqslant$ $\bar{\delta}(n)$ the empty network is not pairwise stable.

The intuition behind Proposition 9 is very simple. Compared to the case of independent signals (in which the empty network is always stable), correlation brings in new incentives to form a link starting from the empty network. Such incentives come from the better inference that linking firms expect to make on the signals and the behaviour of other firms. This better inference comes at no additional cost in terms of revealing one's own signal, and is not present when signals are independent. In order for such new incentives to undermine the stability of the empty network, both the number of firms and the correlation parameter must be large enough. This is intuitive, since they both measure how effective the new inference is, in terms of precision and in terms of number of signals to be inferred. This result makes clear how bilateral sharing agreement may be profitable even in a Cournot oligopoly with homogeneous goods - a conclusion in contrast with the traditional view, well summarized by proposition 4.4 in Raith (1996). The difference in results is mainly due to the difference in the transmission mechanism and in the adopted equilibrium concept. Bilateral contracts allow two firms to reach a sharing agreement without necessarily share their signal with all firms in the market. The pairwise stability concept allow two firms to coordinate their action, possibility that was missing in the early analysis of quid pro quo contracts by Kirby (1988), who looks at the Nash Equilibria of the sharing game, who suffer, in the presence of correlation, of coordination failures. 
The next proposition shows that the instability of the empty network does not come from a general existence problem.

Proposition 10 The complete network is stable for all $\delta \in[0,1]$

Characterizing all pairwise stable networks for this case of correlation is a very hard task. It is actually very difficult to simply understand the effect of correlation of the equilibrium coefficients (and thus on the incentives to form links) in a generic network $g$, as these are expressed in conditions (25)-(26). In order to get some understanding on the effect of correlation, we fully work out the case of four firms, the minimal number that allows for non trivial equilibrium structures.

\subsection{Pairwise Stable Network with 4 Firms}

With four firms, there are eleven architectures to be considered.

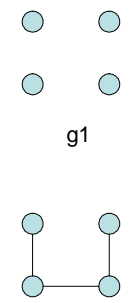

g5

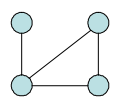

g9
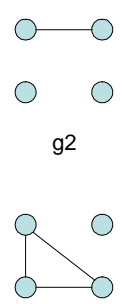

g6

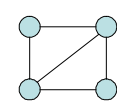

g10
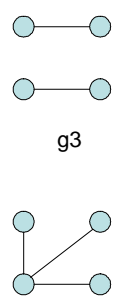

g7

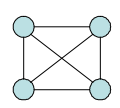

g11
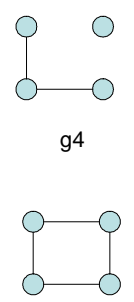

g8

Figure 4. Possible architectures with 4 firms

We identify pairwise stable architectures for various ranges of the correlation parameter $\delta$.

1. When $\delta$ is low $(\delta \leq 0.619)$, the set of stable networks has the same qualitative features as in the case of independent signals: empty and complete networks are stable, plus an ISG network, in particular network "g6" in figure ??. 
2. For an intermediate range of $\delta(0.619 \leq \delta \leq 0.710)$, network "g6" ceases to be stable. In this range, a link between a firm $i$ in the completely connected component and the isolated firm $j$ is formed. It is interesting to note that firm $i$ wishes to form the link for $\delta \leq 0.710$, while firm $j$ wishes to form it for $0.619 \geq \delta$. This highlights the effect of correlation: if correlation is low, then firm $i$ has not a good enough inference on firm $j$ 's signal, and wishes to form the link $i j$; if correlation is high, firm $j$ wishes to form the link, in order to have a good inference on the remaining firms. Note that firm $j$ has a low degree, and would never accept to form the link $i j$ in the absence of correlation.

3. As $\delta$ grows $(0.710 \leq \delta \leq 0.747)$, network "g6" becomes stable again, since firm $i$ has a good enough inference on firm $j$ due to $i$ 's high degree and the high level of correlation.

4. Finally, if $\delta$ grows even more, the empty network ceases to be a stable architecture, as was proved for the general case in proposition 9.

Interestingly, the stability of the network "g6" in the ranges of values $\delta \leq 0.619$ and $0.710 \leq \delta$ results from different incentives of firms. When $\delta$ is low, each of the three firms sharing information would like to link to the isolated firm, whose signal has a large informational value due to the low degree. In this case, the isolated firm decides not to join the group. When $\delta$ is high, the three firms sharing information are excluding the fourth firm, which would like to join if allowed.

\begin{tabular}{|c|c|c|c|}
\hline$\delta \leq 0.619$ & $0.619 \leq \delta \leq 0.710$ & $0.710 \leq \delta \leq 0.747$ & $0.747 \leq \delta \leq 1$ \\
\hline $0 \quad 0$ & 00 & 0 & \\
\hline $0 \quad 0$ & 00 & 00 & \\
\hline g1 & g1 & g1 & \\
\hline g6 & & g6 & g6 \\
\hline g11 & $g 11$ & $g 11$ & $g 11$ \\
\hline
\end{tabular}

Figure 5. Pairwise stable networks for various ranges of $\delta$ 


\section{Conclusions}

We have studied the incentives of oligopolistic firms to share information on a random demand intercept by means of bilateral contracts. We have first studied the benchmark model of i.i.d. signals, for which we have shown that pairwise stable networks are made of fully connected components of increasing size. When linking has a cost, then non complete components can be stable, and in this case density is inversely related to size. We have then shown that when variances of signals are heterogeneous, non regular components of the core-periphery architecture may arise in equilibrium, with high variance firms acting as connectors. Finally, correlated signals introduce new and interesting aspects of analysis, and group formation and exclusion seem to be typical features of this case.

Although we believe that the novelty of our approach brings new and interesting insights in the theory of information sharing, there are several aspects of the present analysis that deserve discussion and improvement. First, the notion of pairwise stability is somewhat specific, and one may wish to study solution concepts that allow multiple links to be formed or severed at the same time. Also, the assumption that information sharing occurs at the ex-ante stage could be removed, so to explore the rich strategic structure of an interim model of information sharing. Finally, the assumption that all firms reveal truthfully their signal is quite strong (although common to all this stream of literature), and the incentives to report false information could be dealt with in an interim model. Finally, a full characterization of stable network in the case of correlated signals is certainly desirable, although technically quite difficult. We leave all of these issues for future research.

\section{References}

[1] Clarke, R., Collusion and the Incentives for Information Sharing, Bell J. Econ. 14 (1983), 383-394.

[2] Gal-Or, E., Information Sharing in Oligopoly, Econometrica 53 (1985), 329-343.

[3] Kirby, A. J., Trade Associations as Information Exchange Mechanisms, Rand J. Econom. 19 (1988), 138-146. 
[4] Jackson, M. O., and A. Wolinsky, A strategic model of social and economic networks, J. Econ. Theory $\mathbf{7 1}$ (1996), 44-74.

[5] Li, L., Cournot oligopoly with information sharing, Rand J. Econom. 16 (1985), 521-536

[6] Novshek, W. and H. Sonnenschein, Fulfilled expectations Cournot duopoly with information acquisition and release, Bell J. Econ. 13 (1982), 214-218.

[7] Radner, R., Team decision problems, Annals Math. Statist. 33 (1962), 857-81.

[8] Raith, M., A General Model of Information Sharing in Oligopoly, J. Econ. Theory 71 (1996), 260-288.

[9] Vives, X., Duopoly information equilibrium: Cournot and Bertrand, J. Econ. Theory 34 (1985), 71-94. 


\section{Appendix}

Proof of Lemma 1. To explicitly derive the term $E\left[\pi_{i}(g)\right]$, consider the best response condition for firm $i$ in the game $\Gamma^{g}$ :

$$
q_{i}=\frac{A+\sum_{j \in N_{i}^{g}} a_{j}+E_{i}\left[\sum_{j \notin N_{i}^{g}} a_{j} \mid a_{i}^{g}\right]-b E_{i}\left[\sum_{j \neq i} s_{j}\left(a_{j}^{g}\right) \mid a_{i}^{g}\right]}{2 b}
$$

which can be rewritten as:

$$
q_{i}=\frac{E_{i}\left[A+\sum_{j \in N} a_{j}-b \sum_{j \in N} s_{j}\left(a_{j}^{g}\right) \mid a_{i}^{g}\right]}{b}=\frac{E_{i}\left[P(a, g) \mid a_{i}^{g}\right]}{b},
$$

where $P(a, g)$ is the market price given realization $a$ in network $g$. Given $a$ and $g$, firm $i^{\prime}$ s equilibrium profits can be expressed as:

$$
\pi_{i}(a, g)=\frac{P(a, g) E_{i}\left[P(a, g) \mid a_{i}^{g}\right]}{b} .
$$

The (interim) expected value of $\pi_{i}(a, g)$ evaluated by firm $i$ in $g$ is:

$$
E_{i}\left[\frac{P(a, g) E_{i}\left[P(a, g) \mid a_{i}^{g}\right]}{b} \mid a_{i}^{g}\right]=\frac{E_{i}\left[P(a, g) \mid a_{i}^{g}\right]^{2}}{b}=b q_{i}{ }^{2} .
$$

Ex-ante expected profits $E\left[\pi_{i}(g)\right]$ of firm $i$ in $g$ are now obtained as follows:

$$
E\left[\pi_{i}(g)\right]=E_{i}^{g}\left[b q_{i}(g)^{2}\right] .
$$

\section{Proof of proposition 1.}

From (31), ex-ante profits are given by $E_{i}\left[b q_{i i}(g)^{2}\right]$. Using standard results, we write for a generic variable $x^{3}$ :

$$
E[x]^{2}=E\left[x^{2}\right]-\operatorname{var}(x)
$$

\footnotetext{
${ }^{3}$ This relation is derived as follows:

$$
\begin{aligned}
E\left[x^{2}\right]-\operatorname{var}(x) & =E\left[x^{2}\right]-E\left[\left(x^{2}+E[x]^{2}-2 x E[x]\right)\right]= \\
2 E[x E[x]]-E[x]^{2} & =E[x]^{2} .
\end{aligned}
$$
}


We can therefore write:

$$
E\left[\pi_{i}(g)\right]=E_{i}\left[b q_{i}(g)^{2}\right]=b E_{i}\left[q_{i}(g)^{2}\right]=b\left(E_{i}\left[q_{i}(g)\right]^{2}+\operatorname{var}\left(q_{i}(g)\right)\right) .
$$

Since from (30) $E_{i}\left[q_{i}(g)\right]=\frac{A}{b(n+1)}$ irrespective of the graph structure, we conclude that the difference between the profits of firm $i$ in graphs $g$ and $g^{\prime}=g-i j$ (for $i j \in g$ ) is the difference in the two variances of equilibrium quantities:

$$
E\left[\pi_{i}(g)\right]-E\left[\pi_{i}\left(g^{\prime}\right)\right]=b\left[\operatorname{var}\left(q_{i}(g)\right)-\operatorname{var}\left(q_{i}\left(g^{\prime}\right)\right)\right] .
$$

We can write down explicit expressions for the equilibrium parameters as follows:To obtain an expression for this difference, let us explicitly derive the equilibrium coefficients $\beta_{i j}$ and $\alpha_{i}$ for this case. Solving conditions (8)-(9) we obtain:

$$
\begin{aligned}
\beta_{i j} & =\frac{1}{b\left(n_{j}^{g}+1\right)}, \forall i \in N_{j}^{g} \text { and } \forall j \in N ; \\
\alpha_{i} & =\frac{A}{b(n+1)} \forall i \in N .
\end{aligned}
$$

It is clear from (35) that the coefficient applied to signal $j$ only depends on the degree of node $j$, and is independent of all other topological properties of the network. Also, the ex-ante expected equilibrium quantity in the aggregate game $\Gamma^{g}$ is $\alpha_{i}$. Using (35)-35), the equilibrium quantity of firm $i$ in game $\Gamma^{g}$ is given by:

$$
q_{i}(g)=\frac{A}{b(n+1)}+\sum_{k \in N_{i}^{g}} \frac{a_{k}}{\left(n_{k}^{g}+1\right) b},
$$

with variance (using the fact that covariance are zero across $a_{i}$ 's and that all variances are equal to $\sigma$ ):

$$
\operatorname{var}\left(q_{i}(g)\right)=\sum_{k \in N_{i}^{g}} \frac{\sigma}{\left(n_{k}^{g}+1\right)^{2} b^{2}}
$$

For $\Gamma^{g^{\prime}}$ we obtain:

$$
q_{i}\left(g^{\prime}\right)=\frac{A}{b(n+1)}+\sum_{k \in N_{i}^{g^{\prime}}} \frac{a_{k}}{\left(n_{k}^{g^{\prime}}+1\right) b}
$$


with variance:

$$
\operatorname{var}\left(q_{i}\left(g^{\prime}\right)\right)=\sum_{k \in N_{i}^{g^{\prime}}} \frac{\sigma}{\left(n_{k}^{g^{\prime}}+1\right)^{2} b^{2}} .
$$

The only two terms that are different in the sums of the above expressions of variances are those that concern signals $j$ and $i$. Using the fact that $n_{i}^{g^{\prime}}=n_{i}^{g}-1$ we obtain that:

$$
\operatorname{var}\left(q_{i}(g)\right)-\operatorname{var}\left(q_{i}\left(g^{\prime}\right)\right)=\sigma\left[\frac{1}{\left(n_{j}^{g}+1\right)^{2} b^{2}}+\frac{1}{\left(n_{i}^{g}+1\right)^{2} b^{2}}-\frac{1}{\left(n_{i}^{g}\right)^{2} b^{2}}\right] .
$$

The conditions for the link $i j$ not to be severed from $g$ are therefore the following:

$$
\begin{aligned}
& \frac{1}{\left(n_{j}^{g}+1\right)^{2}}+\frac{1}{\left(n_{i}^{g}+1\right)^{2}}-\frac{1}{\left(n_{i}^{g}\right)^{2}} \geq 0 \\
& \frac{1}{\left(n_{i}^{g}+1\right)^{2}}+\frac{1}{\left(n_{j}^{g}+1\right)^{2}}-\frac{1}{\left(n_{j}^{g}\right)^{2}} \geq 0 .
\end{aligned}
$$

We now turn to the condition for the link $i j \notin g$ not to be formed. Firm $i$ has an incentive to form $i j$ inducing the network $g^{\prime}=g+i j$ if

$$
\operatorname{var}\left(q_{i}\left(g^{\prime}\right)\right)-\operatorname{var}\left(q_{i}(g)\right)>0
$$

where $q_{i}$ and $q_{i}^{\prime}$ are the equilibrium quantities in $g$ and $g^{\prime}$, respectively. Stability requires that if (42) holds then:

$$
\operatorname{var}\left(q_{j}\left(g^{\prime}\right)\right)-\operatorname{var}\left(q_{j}(g)\right)<0 .
$$

Using again the expression for variances and the fact that $n_{i}^{g^{\prime}}=n_{i}^{g}+1$ we obtain the following condition:

if

$$
\frac{1}{\left(n_{j}^{g}+2\right)^{2}}+\frac{1}{\left(n_{i}^{g}+2\right)^{2}}-\frac{1}{\left(n_{i}^{g}+1\right)^{2}}>0
$$

then

$$
\left(\frac{1}{\left(n_{i}^{g}+2\right)^{2}}+\frac{1}{\left(n_{j}^{g}+2\right)^{2}}-\frac{1}{\left(n_{j}^{g}+1\right)^{2}}\right)<0 .
$$


Proof of Lemma 2: Point $i$ ) is implied by the fact that the RHS of (14) is increasing in $n$ : it is always positive for all $n$. Point ii) is proved along the same lines of point $i$ ). To prove point iii), consider the equations defining $F(n)$ and $f(m)$, respectively:

$$
\begin{gathered}
\frac{1}{n^{2}}-\frac{1}{(1+n)^{2}}=\frac{1}{(1+F(n))^{2}} . \\
\frac{1}{(1+m)^{2}}-\frac{1}{(2+m)^{2}}=\frac{1}{(2+f(m))^{2}} .
\end{gathered}
$$

The LHS of (46) and of (47) coincide for all $m-1$. It follows that also the RHS must coincide for such values, and this happens when $f(n-1)=F(n)-1$.

iv) By definition:

$$
F(n)=\sqrt{\frac{n^{2}(1+n)^{2}}{(1+n)^{2}-n^{2}}}-1 .
$$

For $n=2$ we obtain:

$$
F(n)-F(n-1)=\sqrt{\frac{36}{5}}-\sqrt{\frac{4}{3}}>1 .
$$

We then compute the derivative $\frac{d}{d n}[F(n)-F(n-1)]$, that it is positive for all value $n>2$.

$v$ ) Simple algebraic manipulation of conditions (14)-(15).

vi) Directly from iii) and iv).

Proof of Proposition 2. i) We first show that only regular networks can be pairwise stable. Let $g$ be pairwise stable, and let $h$ be a non regular component of $g$. Then there exist nodes $i$ and $j$ such that $n_{i}>n_{j}$ and $i j \in h$. This means that there exists some node $k \neq j$ such that $i k \in h$ and $j k \notin h$. Pairwise stability of $g$ imposes the following requirements on the degrees of nodes $i, j$ and $k$ :

$$
\begin{aligned}
& n_{k} \leq F\left(n_{i}\right) \text { and } n_{i} \leq F\left(n_{k}\right) \\
& n_{j} \leq F\left(n_{i}\right) \text { and } n_{i} \leq F\left(n_{j}\right) .
\end{aligned}
$$

Note now that since $n_{i}>n_{j}$, then $n_{i}-1 \geq n_{j}$ (remember that degrees are integers). This, together with (48), implies

$$
n_{j} \leq F\left(n_{k}\right)-1 .
$$


Point iii) in lemma 2 together with (49) imply:

$$
n_{j} \leq f\left(n_{k}-1\right) .
$$

We finally use point (ii) in lemma 2 to conclude that:

$$
n_{j}<f\left(n_{k}\right) .
$$

For $g$ to be stable, link $j k$ should not form. This, together with (51), requires that:

$$
n_{k}>f\left(n_{j}\right)
$$

Suppose now that $n_{k} \leq n_{j}$. In this case, condition (48) plus point (iv) in lemma 2 would imply that $n_{i} \leq f\left(n_{j}\right)$, implying, together with (51), that $n_{j}>n_{i}$, a contradiction. We have therefore concluded that $n_{k}>n_{i}$.

This result implies that there exists some node $w$ such that $w k \in h, w i \notin h$ and $n_{w}>n_{k}$. To these three nodes $i, k$ and $w$ we can now apply similar arguments as above to conclude that there exists some neighbour $w^{\prime}$ of $w$ such that $w^{\prime} k \notin h$ and $n_{w^{\prime}}>n_{w}$. Reiterating these steps, and given that the number of nodes $n$ is finite, we obtain a contradiction.

Consider now a regular component of degree $m$. Since $m \leq f(m)$ for all $m$, we conclude that each component of $g$ is fully connected. Components of size 2 are not stable because $F(2)<2$ by point $v$ ) of lemma $(2)$.

ii) The complete network and the empty network are both stable since $F(m) \geq m$ for $m \geq 3$ and $f(1)<1$ by point $v$ ) of lemma 2 . This last inequality also implies that isolated firms do not wish to form a link with firms in a component of size $m \geq 2$. .

iii) Two components of the same size $m \geq 2$ are not consistent with stability, since $f(m) \geq m$ by point $v$ ) of lemma 2 . To complete the proof of point iii), note that any pair of components of sizes $m_{1}<m_{2}$ such that $m_{1} \geq 3, m_{2} \geq 3$ and $m_{2}>f\left(m_{1}\right)$ are such that no link is severed within components nor formed across components.

Proof of Proposition 3:. The change in aggregate profits of isolated firms $i$ and $j$ when they form a link is:

$$
2\left(\frac{\sigma_{j j}}{9}+\frac{\sigma_{i i}}{9}\right)-\frac{\sigma_{j j}}{4}-\frac{\sigma_{i i}}{4}
$$

which is always negative for all values of $\sigma_{j j}$.and $\sigma_{i i}$

Proof of Proposition 5: Consider components $h_{k}$ and $h_{k+1}$, both of the same size $q$. Let $j$ be the firm with highest variance in $h_{k}\left(\sigma_{+}\left(h_{k}\right)=\sigma_{j j}\right)$ and let $l$ be the firm with lowest 
variance in $h_{k}\left(\sigma_{-}\left(h_{k}\right)=\sigma_{l l}\right)$. Stability conditions for component $h$ require that firm $j$ does not sever the link $j l$, where $l$ is the firm with lower variance in $h$, that is, $\sigma_{-}(h)=\sigma_{l l}$ :

$$
\frac{\sigma_{j j}}{m^{2}} \leq \frac{\sigma_{j j}+\sigma_{l l}}{(m+1)^{2}}
$$

Suppose now that there exists firm $i \in h_{k+1}$ such that $\sigma_{i i}<\sigma_{+}\left(h_{k}\right)$. In order for the network $g$ to be stable, firms $j$ must not have an incentive to form a link with firm $i$ :

$$
\frac{\sigma_{j j}}{(m+1)^{2}}>\frac{\sigma_{j j}+\sigma_{i i}}{(m+2)^{2}}
$$

Conditions (53)-(54) imply:

$$
\frac{(m+1)^{2}(m+1)^{2}}{(m+2)^{2} m^{2}}<\frac{\left(\sigma_{j j}+\sigma_{l l}\right)}{\left(\sigma_{j j}+\sigma_{i i}\right)}
$$

If $\sigma_{i i} \geq \sigma_{l l}$ we have that

$$
\frac{(m+1)^{2}(m+1)^{2}}{(m+2)^{2} m^{2}} \leq 1
$$

which is never satisfied. Suppose now that $\sigma_{i i}<\sigma_{l l}$ and that there exists some firm $m$ such that $\sigma_{i i}>\sigma_{l l}$ (if such firm does not exist the consecutiveness of $g$ is not violated). In this case, the same steps used above can be followed by replacing firm $j$ with firm $m$ and firm $l$ with firm $j$

Proof of Proposition 6: We proceed as follows. We first show in step 1 that if $g$ contains a minimal cycle of length at least 4 , then $g$ cannot be pairwise stable for any configuration of variances. We then show that if $g$ is regular and incomplete with degree at least 3 , then $g$ contains a minimal cycle of length at least 4 .

Step 1.. Let $g \neq g^{c}$ be a connected regular network of degree $k$, with $k \geq 2$. Let $P=\{1,2, \ldots x, 1\}$ be a minimal cycle of length $x \geq 4$. For simplicity, in this proof we denote by $\sigma_{i}$ the variance $\sigma_{i i}$. Necessary condition for link $i(i+2)$ not to form is the following for all $i \in\{1,2, \ldots x\}$ :

$$
\sigma_{i+2}>\sigma_{i} \frac{(1+m)^{2}}{2 m+3}
$$

Necessary condition for link $i(i+1)$ not to be severed is the following for all $i \in\{1,2, \ldots x\}$ :

$$
\sigma_{i+1}<\sigma_{i} \frac{m^{2}}{2 m+1}
$$


Given that $\frac{m^{2}}{2 m+1}<\frac{(1+m)^{2}}{2 m+3}$, a necessary condition is that $\sigma_{i}$ be strictly increasing on $\{1,2, \ldots x\}$, from which:

$$
\sigma_{x}>\sigma_{1} \frac{(1+m)^{2}}{2 m+3}
$$

However, necessary condition for link $i x$ not to be severed is the following:

$$
\sigma_{x}<\sigma_{1} \frac{m^{2}}{2 m+1}
$$

It can be easily checked that (57) and (56) are not compatible.

Step 2. Let $g$ be a regular incomplete connected network of degree $k$, with $k \geq 3$. Consider three nodes $i, j$, and $l$ such that $i j \notin g, i l \in g$ and $j l \in g$ (such three nodes must exist since $g$ is not complete and connected). Since $n_{l}^{g}=n_{j}^{g}=k$, there must exist $j_{1} \neq l$ such that $j_{1} \neq i, j_{1} \in N_{j}^{g}$ and $j_{1} \notin N_{l}^{g}$. If $j_{1} \in N_{i}^{g}$, then we obtain the 4-minimal cycle $\left\{i, l, j, j_{1}, i\right\}$ (see figure 6). If, instead, $j_{1} \notin N_{i}^{g}$, consider the set $N_{j_{1}}^{g}$. Again, since $j_{1} \notin N_{l}^{g}$ while $j \in N_{l}^{g}$, and since $n_{j_{1}}^{g}=n_{j}^{g}=k$, there must exist a node $j_{2} \in N_{j_{1}}^{g}$ such that $j_{2} \notin N_{j}^{g}$ (and, therefore, $j_{2} \neq l$ ). If $j_{2} \in N_{l}^{g}$, then we obtain the 4 -minimal cycle $\left\{l, j, j_{1}, j_{2}, l\right\}$. If $j_{2} \notin N_{l}^{g}$ but $j_{2} \in N_{i}^{g}$, then we obtain the 5 -minimal cycle $\left\{i, l, j, j_{1}, j_{2}, i\right\}$. If, instead, $j_{2} \notin N_{l}^{g}$ and $j_{2} \notin N_{i}^{g}$, then consider the set $N_{j_{2}}^{g}$. Since $j_{2} \notin N_{j}^{g}$ and $j_{1} \in N_{j}^{g}$, and since in a $k$-regular network each node has the same degree $k$, there must exist a node $j_{3} \in N_{j_{2}}^{g}$ such that $j_{3} \notin N_{j_{1}}^{g}$ (and, therefore, $j_{3} \neq j$ ). Note also that $j_{3} \neq l, j_{3} \neq i$ by the previous arguments. It follows that if $j_{3} \in N_{i}^{g}$ we obtain a 6 -minimal cycle; a 5-minimal cycle is obtained if $j_{3} \in N_{l}^{g}$; a 4-minimal cycle is obtained if $j_{3} \in N_{j}^{g}$. If none of these is true, then we consider $N_{j_{3}}^{g}$ and repeat the same arguments as above. 


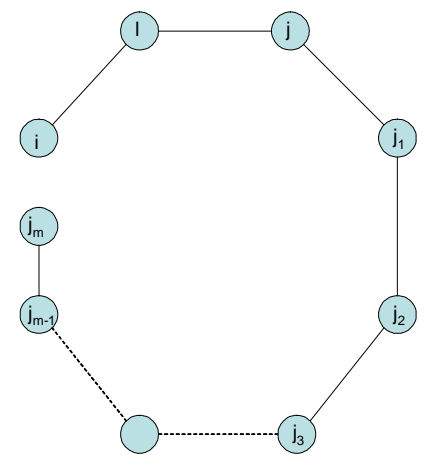

Figure 6

In general, for any $j_{m}$ obtained along this process, there must exist a node $j_{m+1} \in N_{j_{m}}^{g}$ such that $j_{m+1} \notin N_{j_{m-1}}^{g}$. So, if $j_{m+1} \in N_{j_{m-2}}^{g}$, we obtain a 4-minimal cycle; if $j_{m+1} \in N_{j_{m-2}}^{g}$ but $j_{m+1} \in N_{j_{m-3}}^{g}$, we have a 5-minimal cyle, and so on until we check whether $j_{m+1} \in N_{i}^{g}$. If none of these inclusions is verified, we can identify a node $j_{m+2} \in N_{j_{m+1}}^{g}$ such that $j_{m+2} \notin N_{j_{m}}^{g}$ and such that $j_{m+2} \neq j_{m-i}$ for all $i=0,1,2, \ldots, m-1$. Since the number of nodes is finite, the process must stop and a minimal cycle of size at least 4 must be found.

Proof of Proposition 7. Denote by $i \in g_{1}$ and $j \in \cup_{k=2}^{H} g_{k}$. From proposition 1, we know that the following two conditions are necessary and sufficient for the link $i j \in g$ not to be severed:

$$
\begin{gathered}
\frac{\sigma_{i i}}{n^{2}}-\frac{\sigma_{i i}}{(n+1)^{2}} \leq \frac{\sigma_{j j}}{\left(n_{c}+n_{p}+1\right)^{2}} \\
\frac{\sigma_{j j}}{\left(n_{c}+n_{p}\right)^{2}}-\frac{\sigma_{j j}}{\left(n_{c}+n_{p}+1\right)^{2}} \leq \frac{\sigma_{i i}}{(1+n)^{2}} .
\end{gathered}
$$

These conditions can be rewritten as follows:

$$
\begin{gathered}
\sigma_{j j} \geq \sigma_{i i} \frac{(2 n+1)\left(n_{c}+n_{p}+1\right)^{2}}{(1+n)^{2} n^{2}} \\
\sigma_{j j} \geq \sigma_{i i} \frac{\left(n_{c}+n_{p}\right)^{2}\left(n_{c}+n_{p}+1\right)^{2}}{\left(2 n_{c}+2 n_{p}+1\right)(n+1)^{2}}
\end{gathered}
$$

It follows that a link between agents $i \in g_{1}$ and agent $j \in \cup_{k=2}^{h} g_{k}$ is stable only if:

$$
\sigma_{j j} \in\left[\sigma_{i i} \frac{(2 n+1)\left(n_{c}+n_{p}+1\right)^{2}}{(1+n)^{2} n^{2}}, \sigma_{i i} \frac{\left(n_{c}+n_{p}\right)^{2}\left(n_{c}+n_{p}+1\right)^{2}}{\left(2 n_{c}+2 n_{p}+1\right)(n+1)^{2}}\right] .
$$


This condition indirectly imposes an upper bound $r_{\max }$ on the ratio between the larger and the smaller variance of agents in the set $\cup_{k=2}^{h} g_{k}$ :

$$
r_{\max }=\frac{n^{2}\left(n_{c}+n_{p}\right)^{2}}{(2 n+1)\left(2 n_{c}+2 n_{p}+1\right)} .
$$

We now turn to the conditions ensuring that no link between agents $i \in g_{k}$ and $j \in g_{m}$ is formed. Note first that since all peripheral components have the same degree, a necessary condition for $i j$ not to form is that $\sigma_{i i} \neq \sigma_{j j}$. Moreover, from proposition 1 we know that, given that $i$ and $j$ have the same degree, the node with smaller variance has an incentive to form the link; we then need to require that the node with larger variance has no incentive to do so. Without loss of generality, suppose $\sigma_{i}^{2}>\sigma_{j}^{2}$. The link $i j$ does not form if:

$$
\frac{\sigma_{i i}}{\left(n_{c}+n_{p}+1\right)^{2}}-\frac{\sigma_{i i}}{\left(n_{c}+n_{p}+2\right)^{2}}>\frac{\sigma_{j j}}{\left(n_{c}+n_{p}+2\right)^{2}},
$$

yielding:

$$
\sigma_{i i}>\frac{\left(n_{c}+n_{p}+1\right)^{2}}{2 n_{c}+2 n_{p}+3} \sigma_{j}
$$

Therefore, a necessary and sufficient condition for the link $i j$ not to form is the following:

$$
\frac{\sigma_{i i}}{\sigma_{j j}}>\frac{\left(n_{c}+n_{p}+1\right)^{2}}{2 n_{c}+2 n_{p}+3}
$$

Under this condition, the minimum ratio between the larger and the smaller possible variance of peripheral agents is: $r_{\min }=\left(\frac{\left(n_{c}+n_{p}+1\right)^{2}}{2 n_{c}+2 n_{p}+3}\right)^{H-1}$, where $H$ is the number of peripheral planets. Then, a necessary and sufficient condition for the network $g$ to be pairwise stable is that $r_{\min } \leq r_{\max }$, that is condition $(21)$

Proof of Proposition 8. We first prove that for each $C$ there exist integers $m_{1}(C) \leq 4$ and $m_{2}(C) \geq 4$ such that two firms with equal degree $m$ have an incentives to maintain their link if and only if $m_{1}(C) \leq m \leq m_{2}(C)$. Also, for all $m$ in this range, two firms with degree $m-1$ have an incentive to form a link. To see this, note that the stability condition ?? can be rearranged as follows when $n_{i}^{g}=m$ :

$$
\left(\frac{2}{(m+1)^{2}}-\frac{1}{(m)^{2}}\right) \geq C
$$


The LHS of expression (68), when defined on the set of integers, reaches a maximum at $m=4$, and is increasing for $m \leq 3$ and decreasing for $m \geq 4$. The values $m_{1}(C)$ and $m_{2}(C)$ are the first integers above and below the smaller and larger solutions of (68) taken with equality, respectively.

a) Suppose component $h$ is such that $m_{1}(C) \leq n(h) \leq m_{2}(C)$ and is not completely connected. Then, its degree $m$ is strictly less than $m_{2}(C)$. If $m<m_{1}(C)$ then by lemma ?? there exist a firm with an incentive to sever one of its links. If $m \geq m_{1}(C)$, then by lemma ?? we conclude that there exist two firms with an incentive to form a link. This because $m+1 \leq m_{2}(C)$.

b) Suppose that $n(h) \geq m_{2}(C)$ and the degree of $h$ is strictly smaller than $m_{2}(C)$. Lemma ?? implies again that there exist two firms with an incentive to form a link. Suppose the degree of $h$ is strictly larger than $m_{2}(C)$. In this case lemma ?? implies that there are incentives to sever a link

Proof of Proposition 9. Consider the empty network. Applying (25) and (26) we obtain $\alpha=\frac{A}{b(n+1)}$ and $\beta_{i i}=\frac{1+\delta(n-1)}{b[2+\delta(n-1)]}$ where $q_{i}=\alpha+\beta_{i i} a_{i} \forall i$. The payoff is $\pi_{i}=\beta^{2} \sigma$ for all $i$. Now consider two firms, $i$ and $j$, forming a link. Applying (25) and (26) we obtain $\alpha=\frac{A}{b(n+1)}$ for all $i$ and $\beta_{i i}=\beta_{j j}=\beta_{j i}=\beta_{i j}=\frac{2+\delta(2 n-3)-\delta^{2}(n-1)}{b\left[6+3 \delta(n-1)-\delta^{2}(n+1)\right]}$ where $q_{i}^{\prime}=q_{j}^{\prime}=\alpha+\beta_{i i}\left(a_{i}+a_{j}\right)$. Expected profits of firms $i$ and $j$ are $\pi_{i}^{\prime}=\pi_{j}^{\prime}=2 \cdot \beta_{i i}^{2}(\sigma+\rho)$. Now we consider a variable $D=\pi_{i}-\pi_{i}^{\prime}$; when $D$ is positive the empty network is stable, otherwise it is not. By direct computation we get: $D=\sigma\left(\frac{1+\delta(n-1)}{b}\right)^{2} \frac{\left[6+3 \delta(n-1)-\delta^{2}(n+1)\right]^{2}-2(\delta-2)^{2}(\delta+1)[2+\delta(n-1)]^{2}}{[2+\delta(n-1)]^{2}\left[6+3 \delta(n-1)-\delta^{2}(n+1)\right]^{2}}$. The sign of $D$ is the same as the sign of the numerator in the second term. We find that $D$ has only one root in $\delta$ satisfying $D=0$ for all $n \geqslant 4$ and $D$ is strictly positive for $\delta=0$.

Proof of Proposition 10. Consider the complete network. Applying (25) we obtain $\alpha=\frac{A}{b(n+1)}$ and $\beta_{i i}=\frac{1}{b(n+1)}$ where $q_{i}=\alpha+\beta_{i i} \sum_{j \in N} a_{j} \forall i$. The payoff for each firm $i$ is $\pi_{i}=\beta^{2}[n \cdot \sigma+n \cdot(n-1) \rho]$. Now consider two firms, $i$ and $j$, severing a link. Applying (25) we obtain $\alpha=\frac{A}{b(n+1)}$ for all $i$ and $\beta_{i i}=\beta_{j j}=\frac{1+\delta(n-1)}{b\left[n+\delta(n-1)^{2}\right]}, \beta_{i h}=\beta_{j h}=$ $\frac{n[1+\delta(n-1)]}{b(n+1)\left[n+\delta(n-1)^{2}\right]}$ for all $h \neq i, j$, where $q_{i}^{\prime}=\alpha+\beta_{i i} a_{i}+\beta_{i h} \sum_{h \in N /\{i, j\}} a_{h}$. The payoff of agent $i$ id $\pi_{i}^{\prime}=\left[\beta_{i i}^{2}+(n-2) \beta_{i h}^{2}\right] \sigma+\left[2(n-2) \beta_{i i} \beta_{i h}+(n-2)(n-3) \beta_{i h}^{2}\right] \rho$. Now we consider a variable $D=\pi_{i}-\pi_{i}^{\prime}$; when $D$ is positive the complete network is stable, otherwise it is not. By direct computation we get: $D=\sigma \frac{(1-\delta)[1+\delta(n-1)]\{n[n-2+(n-3)(n-1) \delta]-1\}}{b^{2}(n+1)^{2}\left[n+(n-1)^{2} \delta\right]^{2}}$, which is strictly positive for all $\delta \in[0,1)$. 